\title{
Die Haut der Chinesen.
}

\author{
Von \\ Tang Chri und Kuan Chun Woo. \\ Aus dem Anatomischen Institut der Medizinischen Fakultät der \\ Peking Úniversität. \\ (Mit 4 Tabellen). \\ Wir beginnen die histologische Untersuchung mit der Frage: ,Sind \\ Dicke und Verhornungszustände der Epidermis, bei den Chinesen, ebenso \\ wie bei den anderen Rassen and Handteller und Fußsohle am mächtigsten ?"، \\ Durch diese Untersuchungen kann man beweisen, daß bei den Chinesen \\ die Epidermis an der Fingerbeere und den Zehenballen noch dicker als an \\ Handteller oder Fußsohle ist. Sie ist an solchon Stellen beim Menschen \\ schon in der embryonalen Zeit gut ausgewachsen und bezieht sich also nicht \\ bloß auf die ontogenische, sondern auch auf die phylogenische Entwicklung. \\ Mit anderen Worten ist sie nicht nur wegen den mechạnischen Reizungen
} ausgebildet.

\section{Material und Methode.}

Als Material brauchten wir männliche und weibliche Erwachsene, I4 jährigen Knaben, Neugeborenen und s monatigen Embryo. Aus diesen Leichen schnitten wir an 2 I Körperteilen die Hautstücke mit genügendem Unterhautgewebe aus und fixierten sie in Formol, die dann in Paraffin gebettet, in io $\mu$ Längsschnitte zerlegt und mit Hansenschem Haematoxylin. gefärbt wurden. Es wurden folgende Körperteile untersucht :

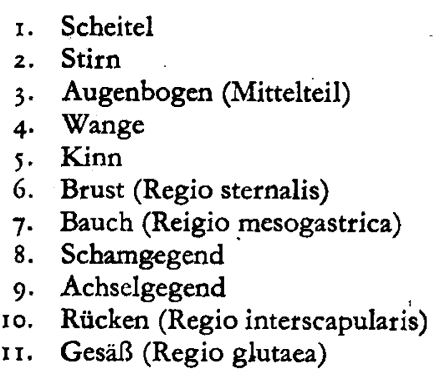

12. Oberarm (laterale Seite, mittlere Höhe)

13. Vorderarm (laterale Seite, mittlere Höhe)

14. Handrücken

I5. Handtellet'

16. Fingerbeere (Regiones volares digitorum)

17. Oberschenkel (Streckseite, mittlere Höhe)

18. Unterschenkel (Streckseite, mittlere Höhe)

19. Fußrücken

20. Fußsohle

2I. Zehenballen (Regiones plantares digitorum pedis) 
Díe Prăparate wurden mit Leizscher Mikroskopie und Mikrometer gemessen. Manch.e davon wurden zuerst projiziert und dann gemessen.

\section{Eigene Befunde.}

\section{Verhornung der Epidermis.}

Aus Tabelle I kann man ersehen, daß der Verhornungszustand der Haut beim Menschen ir der Gegend der Fingerbeere und der Zehenballen am mächtigsten ist.

Beim erwachsenen Mann erschien die Verhornungsstelle der Epidermis an der Fingerbeere am dicksten. Bei der Frau war sie noch dicker als beim Mann. Sie war beim Knaben am Handteller, beim Neugeborenen an der Fingerbeere und Zehenballen und beim Embryo an den Zehenballen am dicksten.

Die nächste relativ dicke Verhornungsstelle fand sich beim Mann an der Fußsohle, bei der Frau an den Zehenballen, beim Knáben an der Fingerbeere, beim Neugeborenen und Embryo an den Zehenballen. Die Stelle, welche von der dünnsten Hornschicht bedeckt ist, war beim Mann der Scheitel, bei der Frau dem Knaben etie Stirn, beim Neugeborenen die Wange und beim Embryo die Wange. Die Untersuchung der sezierten Leichen ergab also, $\mathrm{da} \beta$ sich im allgemeinen die dickste Stelle der Hornschicht an den Fingern, Zehen, Handtellern und Fußsohlen befindet.

\section{Keimschicht der Epidermis.}

Die. Dicke der Keimschicht beim Mann ist an dem Handteller am größten, bei der Frau und dem Knaben an 'der Fingerbeere, beim Neugeborenen am Handteller und beim Embryo an der Schamgegend. (s. Tab. II).

Die gesamte Dicke der Epidermis des erwachsenen Europäers beträgt $0.1 \mathrm{~mm}$ an der Stírn und $0.12 \mathrm{~mm}$ an der Wange (Drosdoff). Nach unserer Untersuchungen ist sie bei den Chinesen wie in Tabelle III aufgeführt.

Die dickste Stelle der Epidermis beim Erwachsenen bemetkt man an der Fingerbeere der Frau (1.258 mm), dagegen die dünnste Stelle am Rücken der Frau $(0.044 \mathrm{~mm})$ und des Mannies $(0.029 \mathrm{~mm})$. Die relative Schwankungsbreife beträgt $62.9: \mathrm{r}$.

\section{Höhe der Papillen.}

Nach Unna (1883) beträgt die Höhe der Lederhautpapillen beim Europäet an der Fußsohle $0.05-0.2 \mathrm{~mm}$. Nach Hoepke fehlt sie am Gesicht, Kinn und Bauch, oder sie ist sehr klein, wie eine Hügel.

Nach unseren Untersuchungen ist sie an der Fingerbeere des Knaben, 
und beim Mann, Neugeborenen und Embryo immer an derselben Stelle am höchsten. Nur bei der Frau an der Fußsohle. Die kleinste Papille beim Mann und der Frau befindet sich an der Rückenstelle, beim Knaben und Embryo an der Wange und beim Neugeborenen an der Brust (Tab. IV).

Die Dicke der Epidermis hängt mit der Höhe der Lederhautpapillen zusammen. Die Stelle, welche dicke Epidermis aufweist, trägt im allgemeinen große Papillen, z. B. am Kopf, Gesicht, Rücken, an der Streckseite der Extremitäten und Rückenseite der Hand oder am Fuß sind sie im allgemeinen dünn und klein, während sie an der Achselgegend, Schamgegend, Handteller, Fußsohle sehr dick und groß hervortreten. Besonders an der Fingerbeere und den Zehenballen wie oben beschrieben (Tab. IV.).

\section{Pigmentkörner der Keimschicht.}

Das Vólumen der Pigmentkörner der Basalzellen der Epidermis hängt mit der Individualität, Rassenzugehörigkeit, Körperstellen, Lichtwirkungen und Behaarungszuständen zusammen (Hoepke). Die Stellen, welche mechanisch gereizt werden, antworten mit vermehrter Pigmentbildung (Kyrle). Miescher hat betont, daß das sich ablösende Haar Pigment an die Papille abgibt. Nach Hoepke sind es bei einem starken Pigmentgehalt immer erhebliche Haare: Man kann also annehmen, daß das Pigment sich auf das Vorhandensein der Haare bezieht. Aber nach unserer Untersuchungen ist dieses bei den Chinesen nicht die Regel, z.B, enthalten bei der Frau die Basalschicht der Scheitelhaut und Achselhaut sehr wenig Pigment. Demgegenüber besitzt die Wangenhaut bei denselben Individuen reichlich Pigment. Beim Mann treten die Pigmentkörner sehr häufig in der Brust und Gesäßhaut hervor; bei Chinesen ist die erstere dünn behaart und ohne lange Terminalhaare (Tang-chi). Besonders ist die Gesäßhaut bei beiden Geschlechtern ausgezeichnet pigmentiert. Ferner befindet sich am 'Handteller im allgemeinen spärlich Pigment. Die Fingerbeere und Fußsohle sowie die Zehenballen sind im allgemeinen frei von Pigment.

Die Pigmentkörner der Epidermis treten nicht nur in den Basalzellen hervor, sondern sie verbreiten sich auch bis zur mittleren Schicht der Stachelzellen der Epidermis. Sie sind kornförmig von hellbraunem bis dunkelbraunem Ton, und besetzen im allgemeinen mehr die äußeren Seiten als die inneren der Zellen: In der Umgebung der Zellkerne findet sich eine helle Plasmazone frei von Pigment. Diese Zone erscheint beim Embryo und Neugeborenen breiter als bei Erwachsenen.

Die Granulazellen der Epidermis bei Erwachsenen sind an der Hand und dem Fuß sehr groß und liegen in 3-4 Reihen parallel an der Oberfläche der Stachelzellen. Beim Neugeborenen und Embryo sind an denselben 
Stellen solche Zellen plattenförmig und stehen nur in I-2 Schichten. Beim Embryo kann man in der Scheitelhaut, Stirn und Augenbogenhaut keine solche Zellen wahrnehmen.

\section{Zusammenfassung.}

i. Das Volumen der Pigmentkörner der Epidermis hängt mit den Behaarungszuständen und der Lichtwirkung wahrscheinlich nicht zusammen. Es steht nach unserer Untersuchung zu der Individulität, den Altersstufen und Körperstellen in Beziehung.

2. Der Verhornungszustand bei den Chinesen ist an der Fingerbeere und den Zehenballen am mächtigsten.

3. Die dickste Stelle der Epidermis der erwachsenen Chinesen beträgt durchschnittlich an der Fingerbeere $1.258 \mathrm{~mm}$ und die dünnste Stelle am Rücken $0.029-0.044 \mathrm{~mm}$.

4. An der Stelle, die von einer dicken Epidermis bedeckt ist, bemerkt man immer große Papillen in der Lederhaut, z. B. an der Achselgegend, Schamgegend, Fingerbeere, Zehenballen, u. s. w.

\section{Schrifttum :}

I) Tang-chi: Quantitative Untersuchung der Anhangsorgane der Haut bei einem Chinesen (Mitteilung der med. Fakult der staatl. Univ. Peking. Bd. I. H. I. 1939).

2) Tang-chi: Die Sexualhaare der Chinesen (Mitteilung d. med. Fakult. d. staatl. Univ. Peking. Bd. 3. H. 4. 1942).

3) Apolant: Uber den Verhornungsprozeß (Arch. f. mikrosk. Anat. Bd. 57. S. 766-798. I901).

4) Blaschko: a) Beitrag zur Anat. d. Oberhaut. (Arch. f. mikr. Anat. Bd. 30. S. 495529. 1887 ).

5) Semon: Die Fußsohle des Menschen (Arch.'f. mikr. Anat. Bd. 82. 1903).

6) Studnicka: Vergleichende Untersuchungen über Epidermis der Vertebraten, "Anat. Hefte, abt. I. Bd. 39, S. 1-269. 1090).

7) Miescher: Die Chromatophoren in der Haut des Menschen. (Klin. Wochenschr. Bd. I. Nr. 3. 1922).

8) Hoepke: Die Haut. (Handbuch der mikroskop. Anat. des Menschen.-Möllendorff. III/I I927).

9) Fischel: Zur Pigmententwicklung. (Anat. Anz. Bd. 12. 1896).

ro) Rudolf Martin: Lehrbuch d. Anthropologie II. Auf. 1928. 
OKAJIMAS FOLIA ANATOMICA JAPONICA, BD. 22.

TAF. XVII.

Tabelle I. Verhornungszustände der Epidermis.

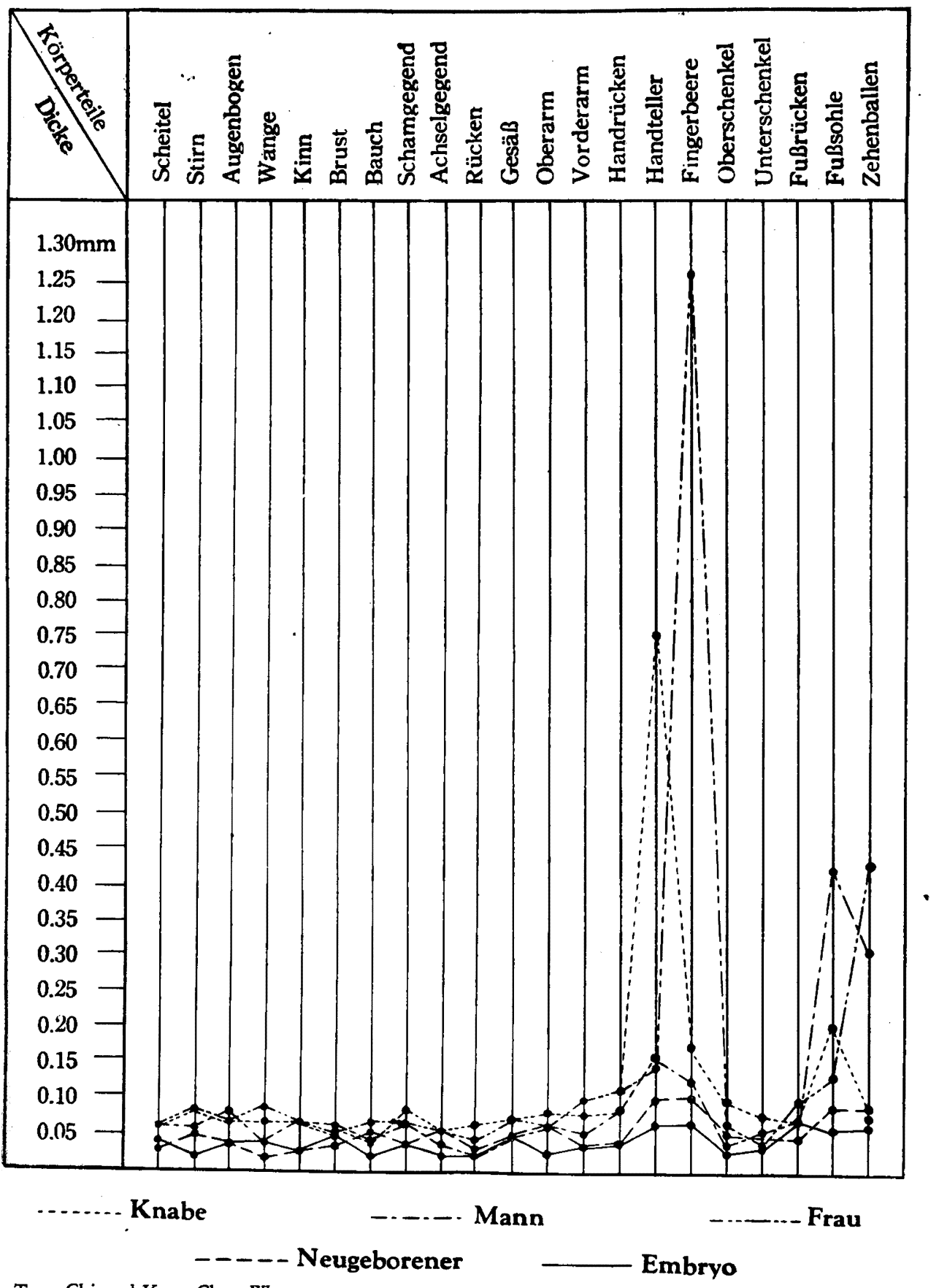

Tang Chi und Kuan Chun Woo. 
OKAJIMAS FOLIA ANATOMICA JAPONICA, BD. 22.

TAF. XVUI.

Tabelle II. Dicke der Keimschicht der Haut.

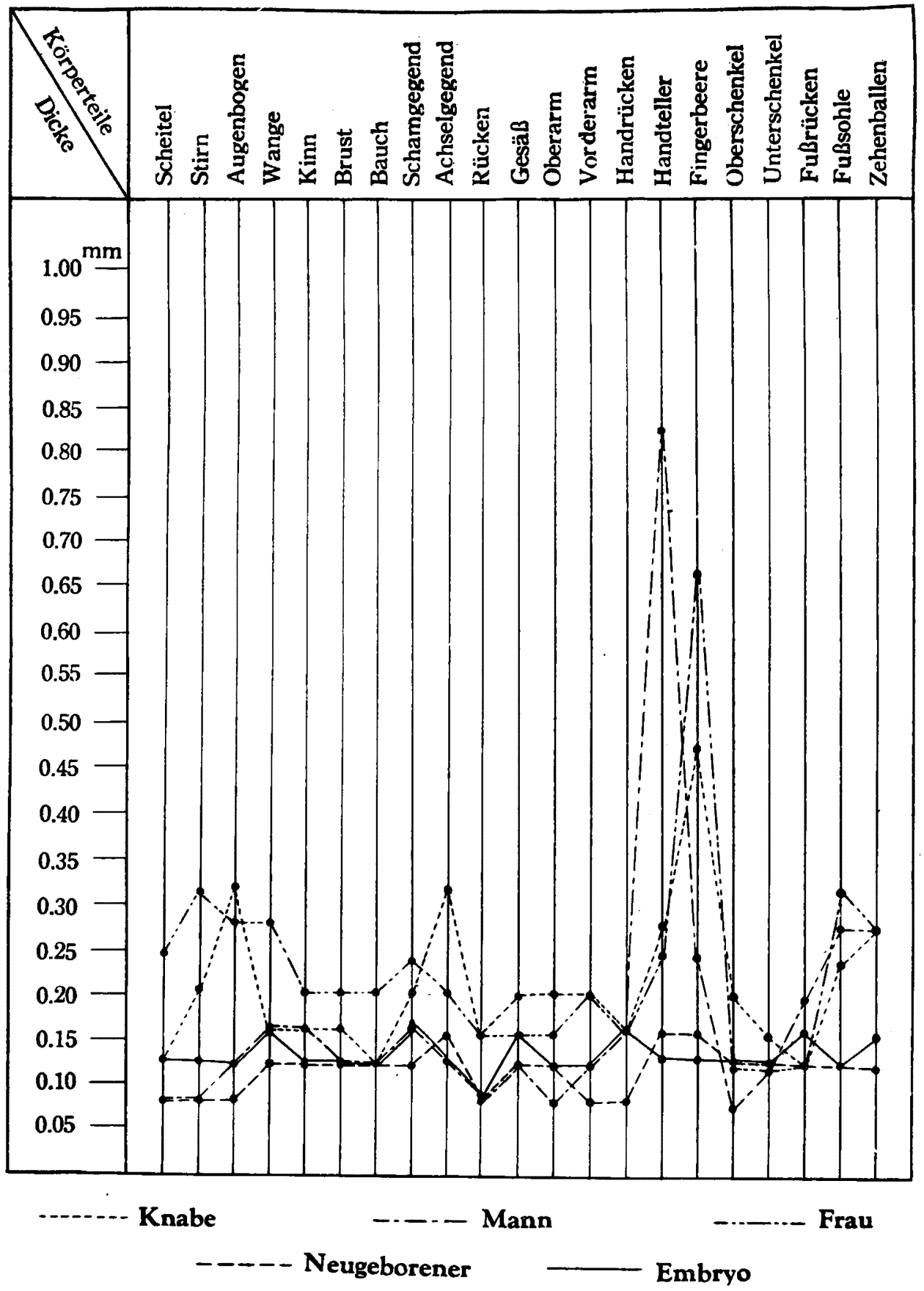

Tang Chi und Kuan Chun Woo. 
OKAJIMAS FOLIA ANATOMICA JAPONICA, BD. 22.

TAF. XIX.

Tabelle III. Gesamte Dicke der Epidermis (durchschnittlich).

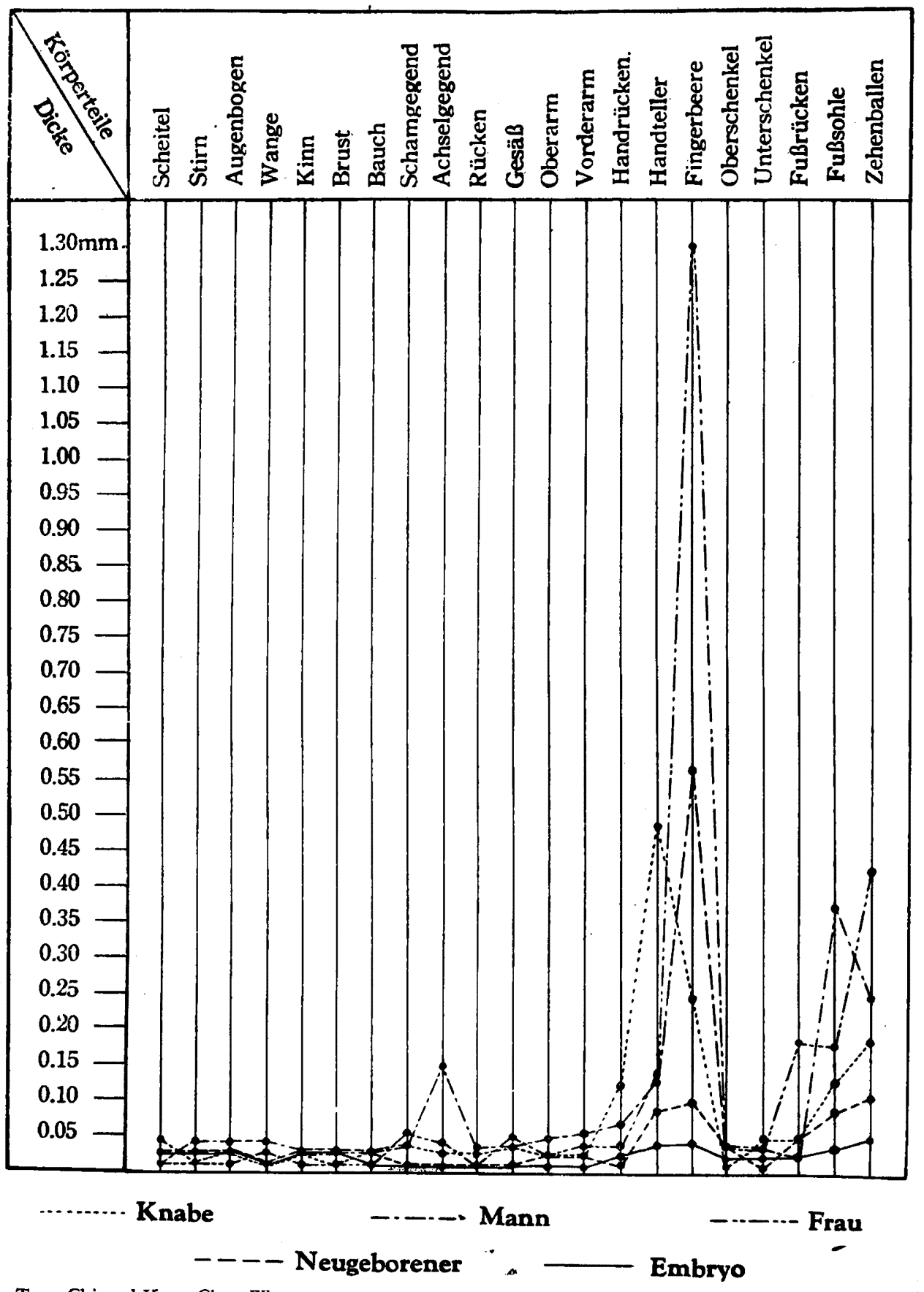

Tang Chi und Kuan Chun Woo. 
OKAJIMAS FOLIA ANATOMICA JAPONICA, BD. 22.

TAF. XX.

Tabelle IV. Höhe der Papillen der Lederhaut.

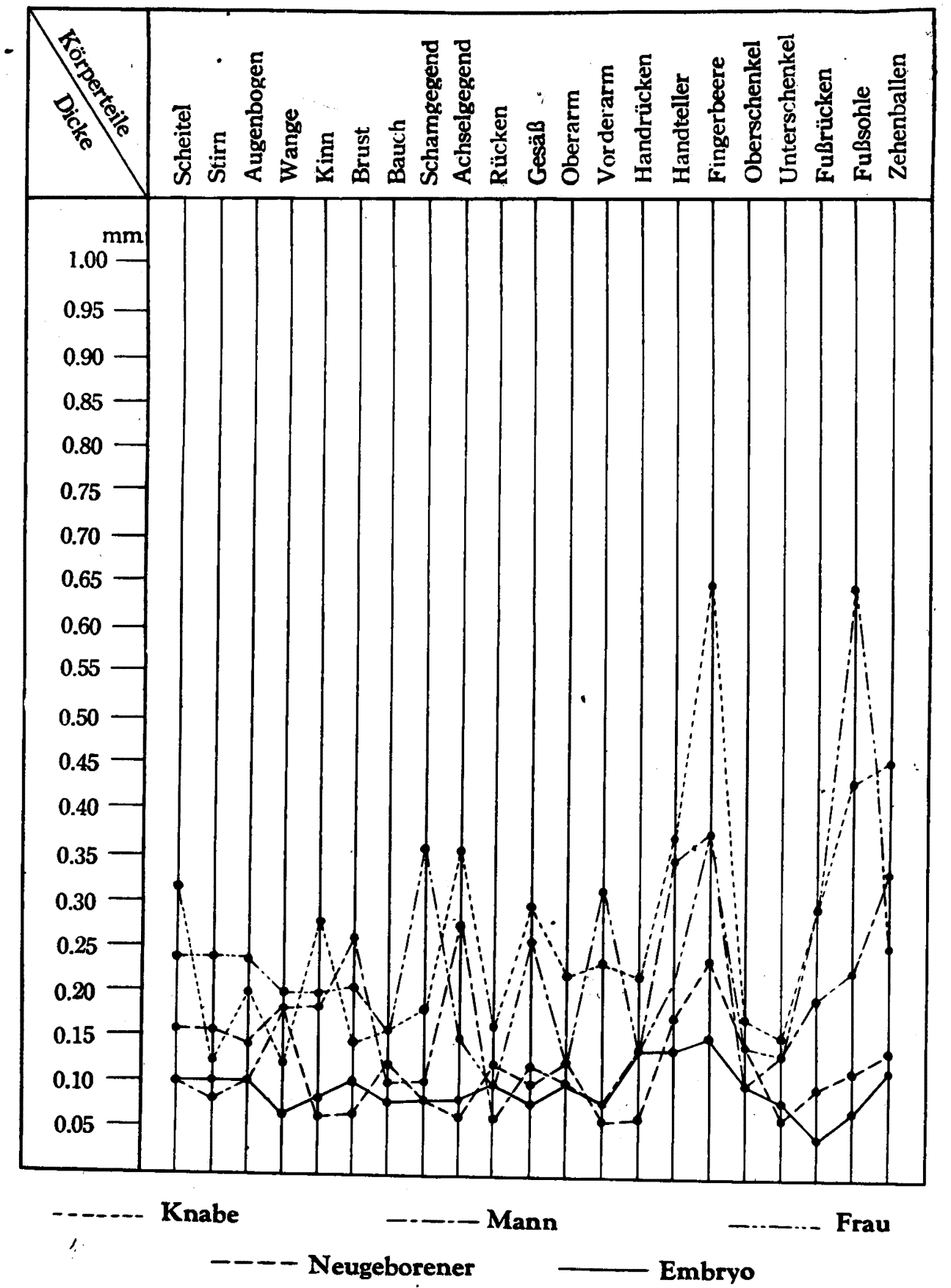

Tang Chi und Kuan Chun Woo. 